$\xi=-1$

\title{
Fully Convolutional Neural Network for Malaysian Road Lane Detection
}

\author{
N. J. Zakaria ${ }^{1,2}$, H. Zamzuri ${ }^{1,2}$, M. H. Ariff ${ }^{1,2}$, M. I. Shapiai ${ }^{1}$, S. A. Saruchi ${ }^{1,2}$, N. Hassan ${ }^{1,2 *}$ \\ ${ }^{\text {I} F a c u l t y ~ o f ~ M a l a y s i a-J a p a n ~ I n t e r n a t i o n a l ~ I n s t i t u t e ~ o f ~ T e c h n o l o g y, ~ U n i v e r s i t i ~ T e k n o l o g i ~ M a l a y s i a, ~} 54100$ Kuala Lumpur, Malaysia \\ ${ }^{2}$ Vehicle System Engineering Ikohza, Universiti Teknologi Malaysia, 54100 Kuala Lumpur, Malaysia \\ *Corresponding author E-mail: jannahzakaria94@gmail.com
}

\begin{abstract}
Recently, a deep learning, Fully Convolutional Neural Network (FCN) has been widely studied because it can demonstrate promising results in the application of detection of objects in an image or video. Hence, the FCN approach has been proposed as one of the solution methods in mitigating the issues pertinent to Malaysia's road lane detection. Previously, FCN model for lane detection has not been tested in Malaysian road conditions. Therefore, this study investigates the further performance of this model in the Malaysia. The network model is trained and validated using the datasets obtained from Machine Learning NanoDegree. In addition, the real-time data collection has been conducted to collect the data sets for the testing at the highway and urban areas in Malaysia. Then, the collected data is used to test the performance of the FCN network in detecting the lane markings on Malaysia road. The results demonstrated that the FCN method is achieving $99 \%$ of the training and validation accuracy.
\end{abstract}

Keywords: Fully Convolutional Neural Network (FCN); lane detection; deep learning.

\section{Introduction}

An intelligent vehicle with the implementation of an intelligent system such as an Advanced Driver Assistant System (ADAS) is designed to assist the driver in improving the driving process and vehicle collision. Correspondingly, lane detection is a part of the intelligent vehicle systems which can be used in ADAS [1]. Lane detection can be defined as the operation to display the location of the lane markers on the road into an intelligent system [2]. It is a challenging task due to the variety of road and weather conditions while driving.

The conventional method of lane detection mostly utilises a handcrafted technique to extract the features. The extraction is a better technique for the network's training, yet it is time-consuming. While, the deep neural networks do not necessary for the implementation of hand-crafted features combined in a model-based approach. In contrast, a deep neural network does not require the implementation of hand-crafted features. It is because during training, the network is able to learn the features representation from the input images [3]. Practically, some approaches used the point cloud/top view images as the training dataset which produced clear and uncluttered views of the lane images with minimum noise. However, this camera configuration only yields the optimum view of the lane markings. Thus, this study used a frontfacing camera to perform the lane detection as it can capture the whole front facing scene, including all possible, clutters. This step provides more information about the road scene images for the network to learn.

Over the decades, several deep learning methods for lane detection had been utilised such as Siamesed Fully Convolutional Networks (s-FCN-loc) based on the VGG-net architecture [4]. The authors focused on exploiting RGB-channel images, and semantic contour maps. Next, in [3] presented an implementation of a deep neural network to estimate the lane positions that operates on images from laterally-mounted down-facing cameras. Subsequently, previous researchers performed road detection using point cloud topview images using only Light Detection and Ranging (LIDAR) data. Other than that, there have also been a variety of conventional image processing methods utilised in road lane detection such as Hough Transform [5, 6], Kalman Filter [7], Vanishing Point [5, 8], Edge Detection [9], etc. In [5] proposed a combination of the Hough Transform and the Vanishing Point technique in highway lane detection. It is succeeded in the implementation of road triangle extraction, the road-width calculation for scale factor determination and the Region of Interest (ROI) for detection of the road boundary. In [6] also proposed the same method with the addition of a comparative study between the Standard Hough Transform (SHT) and the Randomized Hough Transform (RHT).

This paper proposes a Fully Convolutional Neural Networks (FCN) method in detecting the lane markings on Malaysian roads. It took the arbitrary size of the input road lane images and detected the pixel-wise version of this images by using a semantic segmentation process. It is also consisted of extracting and detecting the lane features in a pixel of the images. Even though previous studies, to the best of our knowledge, it has not been tested under Malaysian road conditions yet. Therefore, the main contribution of this paper is to propose the extraction of the full-scale features for semantic segmentation using the existing FCN model and to further investigate the performance of the model by testing the network under Malaysian road conditions. The training datasets were obtained from the Machine Learning NanoDegree [10]. The datasets for the network's testing process consisted of various type of road conditions collected from the real-time data collection. The results showed that the FCN method can indeed detect the target lane on the road.

This paper is structured as follows: In Section 2, the methodology that applied in this study is explained. Then, the experimental and 
modeling results are discussed in Section 3. Finally, the conclusions and the future works are drawn in the last section.

\section{Methodology}

\subsection{Datasets}

The training and validation datasets were obtained from the Machine Learning Nanodegree [10]. The input image datasets were RGB images with 720 pixels on the $y$-axis and 1280 pixels on the $\mathrm{x}$-axis, with a frame rate of 30 (frames per second) fps. The variability of the road conditions in the datasets were varied in term of time, places and weather. Thus, the datasets contained images with the scenes of rainy and cloudy days, traffic, curvy roads, intersections, and constructions areas.

On the other hand, for the testing datasets, the data acquisition was acquired from the forward-facing camera. As shown in Figure 2, the e-CAM21_CUMI1290_MOD camera model produced RGB images with $30 \mathrm{fps}$ and $270 \mathrm{p}(1280 \times 720)$ HD pixels of dimensions [11]. The camera is mounted on the rear-view mirror inside the vehicle, so that it can capture the highway and urban areas with variability in the environmental conditions.

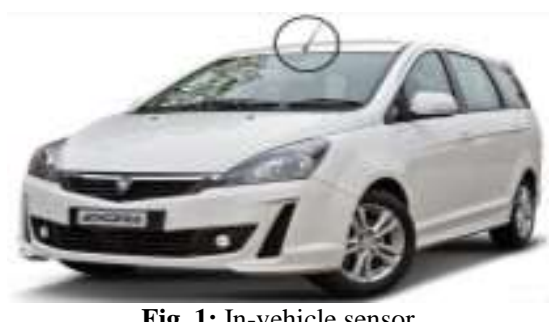

Fig. 1: In-vehicle sensor

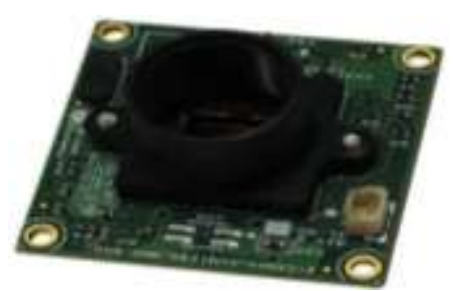

Fig. 2: e-CAM21_CUMI1290_MOD camera models

\subsection{Fully Convolutional Neural Network (FCN) Archi-} tecture

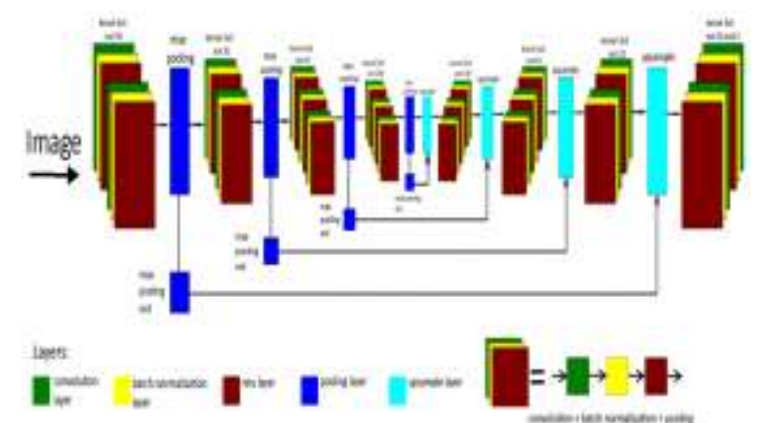

Fig. 3: Fully Convolutional Neural Network Architecture

FCN is most commonly utilised to detect information regarding images. The main advantage of FCN is that it has demonstrated promising results in terms of accuracy of performance. The architecture of FCN is per shown in Figure 3 [12]. According to the figure, FCN consisted of several layers which are convolutional, max pooling, normalization, dropout, deconvolutional, and unpooling layers. FCN emphasises the requirement of the target object's shape and location by using a segmented image, which is taken from the original image. The input layer consists of raw
RGB data images with dimensions of $1280 \times 720$ HD pixels. Every single image has its own mask. Mask is a monochrome image, whereby each group of the objects in the images is determined by a particular range number of colour [12]. The colour range for images is basically from 0 until 255 . In this study, the images are divided into two groups which are lane and background.

Firstly, the input image consists of the raw data image of the lane road is inserted into the FCN network. The next stage is to go through the image pixel by pixel and compare with the masking image (expected output) to determine, whether they are match or otherwise. In the first layer which is the convolutional layer, the image is filtered using a kernel filter to find the specific feature of the desired output. It contains 64 filters with 7 strides. The next convolutional layer had a filter with the size of approximately half of the previous convolutional layer.

The resulting image then goes through the pooling layer to shrink the image size. The pooling layer that applied in this network was $2 \times 2$ with $3 \times 3$ of a kernel size. The size shrinking process is important to reduce the computational time of the network. The normalization and dropout layer are added to increase the accuracy of the network, while the unpooling and the deconvolutional layers are to resize the processed image back to its initial size. The activation function consisting of Rectified Linear Units (RELU) is applied in the network model. In addition, the dropout is also applied to the network with the value of 0.2 , so as to overcome the overfitting problem. The arbitrary dropout turns off a fraction of the neurons during the training step and decreases the dependency on the training set by a certain amount. The input images pass all the network layers one after another and the resulting output is compared with its mask image using the loss function.

Subsequently, the weights were updated to the optimum values required by the network. The last process was the testing operation to evaluate the accuracy of the detection in the network. In addition, the data that was used in the testing process was not included in the training dataset. Last but not least, the network was evaluated based on the accuracy and loss of the training and validation of the network.

\section{Results and Discussion}

The proposed network was trained using open source Tensor flow from the deep learning library with a batch size of 64 and 10 epochs. The training and validation datasets were obtained from the Machine Learning Nanodegree [10]. The datasets for testing were acquire from a real-time data collection using a camera mounted above the rear-view mirror of the vehicle and pointing forwards. Both datasets had a resolution of $720 \times 1280$ pixels. The target scenarios of the dataset included highway-night, urban-night, highway-day, and urban-day in Malaysia. Table 1 presents the detection accuracy and loss of training and validation of the network model. The training and validation accuracy both obtained almost the same value, which was $99 \%$ with a training loss is 0.0323 and validation loss of 0.0278 .

Table 1: Results of training and validation

Table 1: Results of training and validation
\begin{tabular}{|c|c|c|}
\hline & Accuracy & Loss \\
\hline Training & 0.9900 & 0.0323 \\
\hline Validation & 0.9922 & 0.0278 \\
\hline
\end{tabular}

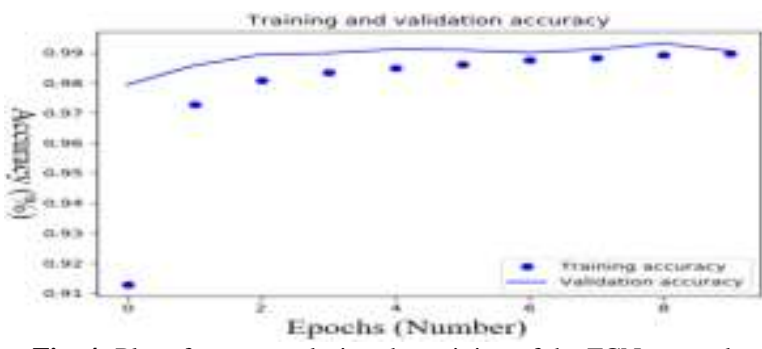

Fig. 4: Plot of accuracy during the training of the FCN network 


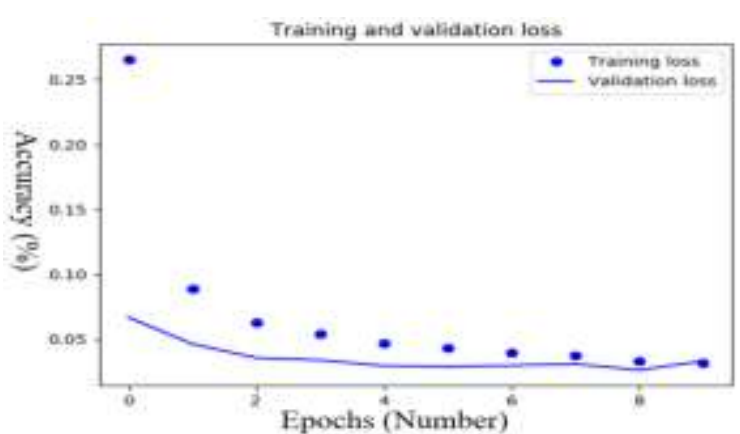

Fig. 5: Plot of loss during the training of the FCN network

Figure 4 and Figure 5 show that the training accuracy and training loss were synchronised with the validation accuracy and validation loss. Therefore, the model was not overfitting as there was not much gap between the training and validation accuracy even though the validation loss and validation accuracy were non-linear. In addition, the network model performance was capable of lane detection since there was a slight loss between the validation loss and the training loss.

Therefore, even though only using the datasets from MLND for training and validation and the datasets of the Malaysian roads for testing only, the network was still can found to perform well. It was able to detect the Malaysian road lane markings even though the network had not learned or trained for the Malaysian road conditions. Hence, the Fully Convolutional Neural Network model was found capable of detecting the road lanes in Malaysia. Figure 6 (a) to Figure $6(\mathrm{~g})$ present the lane detection results for a variety of lighting and road conditions. This figure shows the detected lanes of the road which are represented as a green surface colour.

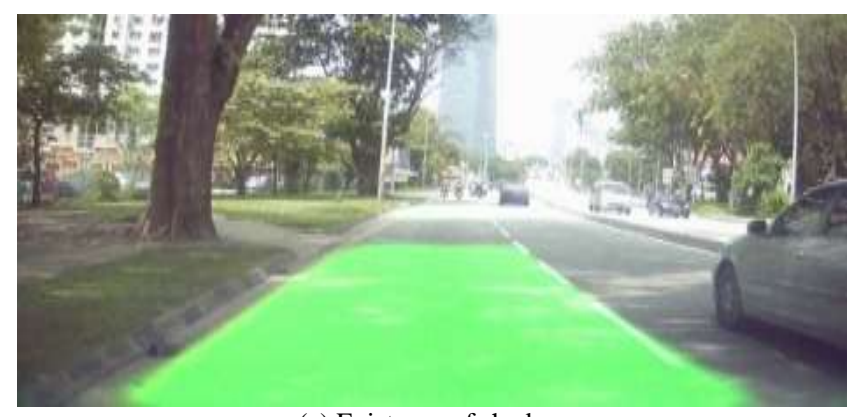

(a) Existence of shadow

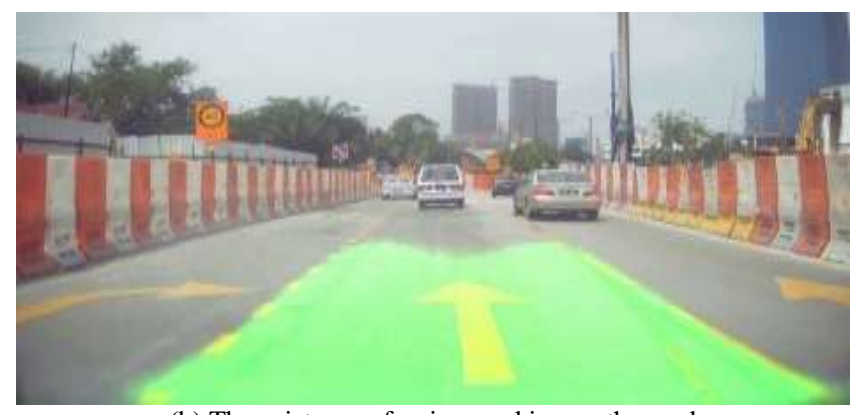

(b) The existence of a sign marking on the road

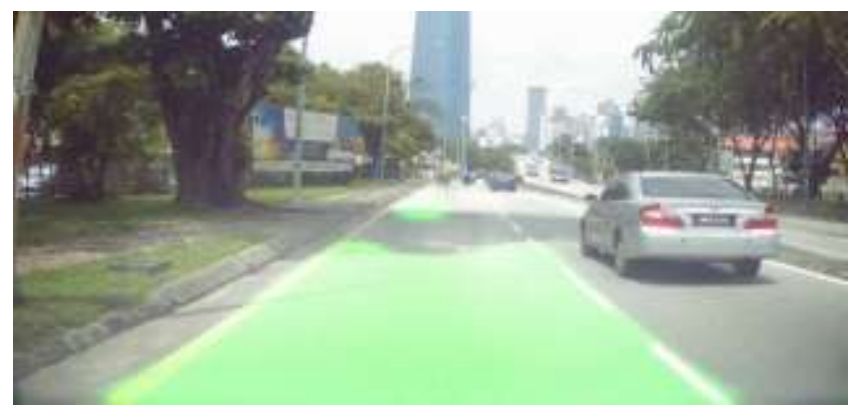

(c) Vehicle overtaking

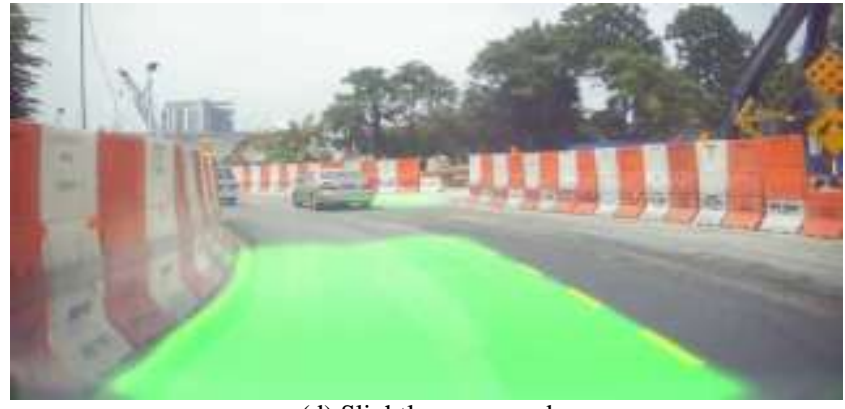

(d) Slightly curve road

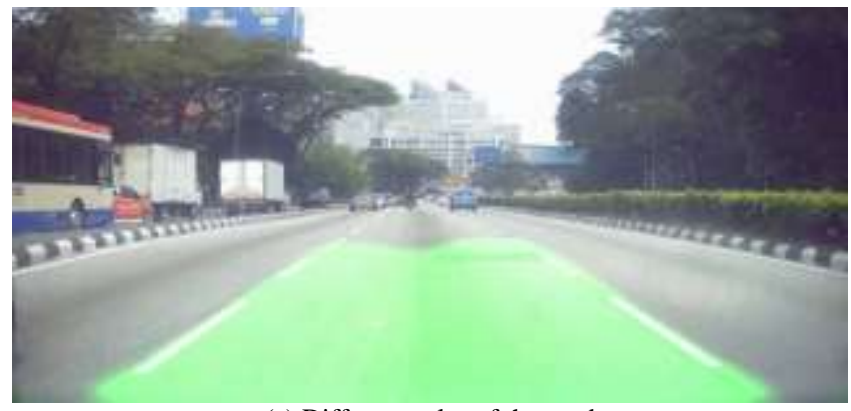

(e) Different color of the road

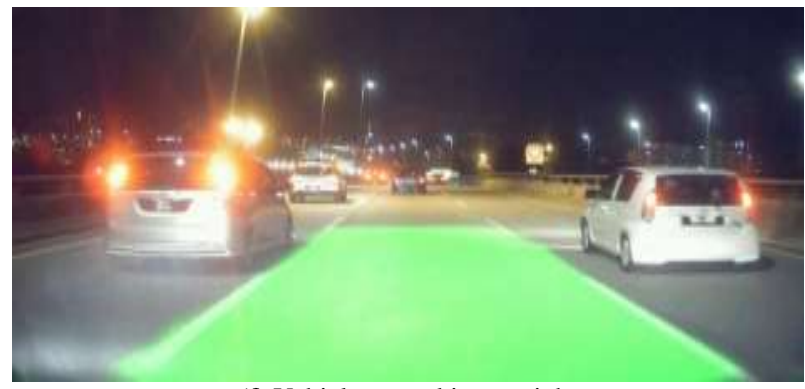

(f) Vehicle overtaking at night

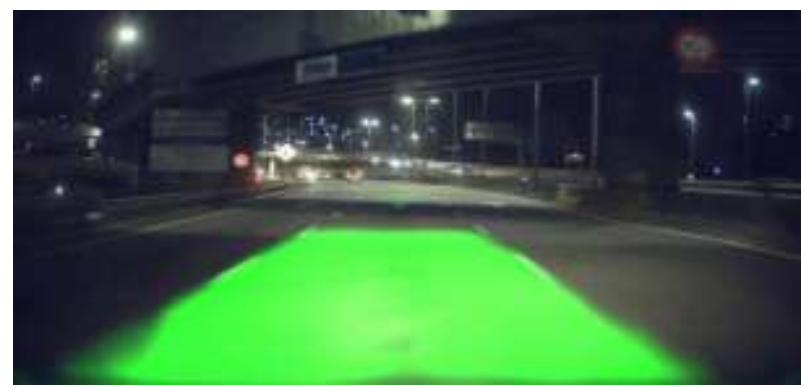

(g) The existence of shadow at night

Fig. 6: Lane detection results. (a) The existence of shadow (b) The existence of a sign markings on the road (c) Vehicle overtaking (d) Slightly curved road (e) Different colour of the road lane (f) Vehicle overtaking at night (g) The existence of shadow

Based on the results, the validation and training loss found by this study were larger than the previous study as depicted in Table 2. As stated by [10], the model has some weaknesses including losing the right side of the lane at the one point. In addition, even though this model has given promising results when testing on actual Malaysian roads, further experiment and collection of data needs to be added to improve the model accuracy. In the opinion of the authors, the accuracy also can be further enhanced by combining the current FCN with an image processing method.

Table 2: The performance comparison of the proposed study with the previous work

\begin{tabular}{|c|c|c|}
\hline & $\begin{array}{c}\text { FCN With Malaysia's } \\
\text { Road Lane Testing Da- } \\
\text { taset }\end{array}$ & $\begin{array}{c}\text { FCN Without Malay- } \\
\text { sia's Road Lane Testing } \\
\text { Dataset }\end{array}$ \\
\hline Training loss & 0.0323 & 0.0046 \\
\hline Validation loss & 0.0278 & 0.0048 \\
\hline
\end{tabular}




\section{Conclusion}

This paper presents a deep learning approach namely Fully Convolutional Neural Network (FCN) for Malaysia road lane detection. The training and validation datasets during the modeling process are taken from Machine Learning NanoDegree (MLND), while the testing datasets are obtained from the raw images of Malaysia road lane. The ability of FCN model in detecting Malaysia road lane mark is evaluated according to the training and validation accuracies, and training and validation losses. The model achieved $99 \%$ of accuracy for both training and validation. Moreover, the network produced 0.0323 and 0.0278 of training and validation losses. These results proved that the FCN network model is able to detect the Malaysian road lane, even though the network has not been learned or trained with the Malaysia road conditions. In future work, for further enhancement of the network model, the combination with other methods such as image processing in the modeling process is suggested.

\section{Acknowledgement}

The work presented is funded by the Ministry of Higher Education Research University Grant, under Potential Academic Staff Grant Universiti Teknologi Malaysia (vote no: PY/2017/01140).

\section{References}

[1] Tian, Y., Gelernter, J., Wang, X., Chen, W., Gao, J., Zhang, Y., \& $\mathrm{Li}, \mathrm{X}$. (2018). Lane marking detection via deep convolutional neural network. Neurocomputing, 280, 46-55.

[2] Kaur, G., \& Kumar, D. (2015). Lane detection techniques: A review. International Journal of Computer Applications, 112(10), 4-8.

[3] Gurghian, A., Koduri, T., Bailur, S. V., Carey, K. J., \& Murali, V. N. (2016). Deeplanes: End-to-end lane position estimation using deep neural networks. Proceedings of the IEEE Conference on Computer Vision and Pattern Recognition Workshops, pp. 38-45.

[4] Wang, Q., Gao, J., \& Yuan, Y. (2018). Embedding structured contour and location prior in siamesed fully convolutional networks for road detection. IEEE Transactions on Intelligent Transportation Systems, 19(1), 230-241

[5] Ali, M., Radzi, A., \& Saad, H. M. (2017). A new approach to highway lane detection by using Hough Transform technique. Journal of ICT, 16(2), 244-260.

[6] Mongkonyong, P., Nuthong, C., Siddhichai, S., \& Yamakita, M (2018). Lane detection using Randomized Hough Transform. IOP Conference Series: Materials Science and Engineering, 297(1), 111.

[7] Lee, Y., \& Kim, H. (2016). Real-time lane detection and departure warning system on embedded platform. Proceedings of the IEEE 6th International Conference on Consumer Electronics, pp. 1-4.

[8] Zhang, Y., Su, Y., Yang, J., Ponce, J., \& Kong, H. (2018). When Dijkstra meets vanishing point: a stereo vision approach for road detection. IEEE Transactions on Image Processing, 27(5), 21762188.

[9] Narote, S. P., Bhujbal, P. N., Narote, A. S., \& Dhane, D. M. (2018) A review of recent advances in lane detection and departure warning system. Pattern Recognition, 73, 216-234.

[10] Virgo, M. (2017). Lane detection with deep learning. https://github.com/mvirgo/MLND\%Capstone/blob/master/MLND \%20Capstone\%20Project\%20Report.pdf.

[11] E-con Systems India Pvt. Ltd. (2017). Camera. eCAM21_CUMI1290_MOD datasheet.

[12] Ozhiganov, I. (2016). Using fully convolutional neural networks. https://www.azoft.com/blog/fully-convolutional-neural-networks/. 